\title{
Obstructive Sleep Apnea and Prolonged Mechanical Ventilation
}

Patients with prolonged mechanical ventilation have a high one year mortality, with recognized contributing factors including COPD, cardiac failure, renal failure, and respiratory muscle weakness. ${ }^{1}$ The study by Diaz-Abad et $\mathrm{al}^{2}$ shows that obstructive sleep apnea (OSA) is very common among selected obese patients who have weaned from prolonged mechanical ventilation. The study raises as many questions as it answers. Did OSA significantly contribute to the need for prolonged mechanical ventilation? Is OSA common among non-obese patients with prolonged mechanical ventilation? What is optimal evaluation for OSA among ventilator dependent and recently weaned patients? With OSA common among obese patients weaned from prolonged mechanical ventilation, could it have been diagnosed sooner? If CPAP or bi-level PAP had been started sooner, could the patients have come off mechanical ventilation sooner and been decannulated sooner?

\section{See the Original Study on Page 229}

Rather than wait until a patient is entirely off mechanical ventilation to evaluate for OSA, why not evaluate as soon as the patient is ventilator-free throughout the day. Bedside studies such as overnight oximetry while the tracheostomy tube is capped can screen for OSA. Tracheostomy patients with suspected OSA on screening studies can then be managed with CPAP or bi-level PAP given via nasal or face mask while using a speaking valve. Using a speaking valve during PAP therapy allows easy inspiratory flow should the mask come off or if the PAP pressure is not high enough to eliminate obstruction on all breaths. Bedside limited channel sleep studies can be used instead of polysomnography to diagnose OSA and qualify patients for home CPAP, and bedside auto-CPAP or oximetry study on CPAP can help determine CPAP levels needed.

The study also raises issues of optimal tracheostomy tube management. They evaluated patients for decannulation with direct laryngoscopy. In our experience, tracheostomy tube manometry can guide whether a patient needs downsizing or is ready for speaking valve or capping, with laryngoscopy needed only for patients with elevated manometry after downsizing. ${ }^{3}$ Leaving patients with open tracheostomy tubes at night can interfere with communication and secretion clearance. In our experience, for patients with good manometry capped, capping is well tolerated at night in patients without OSA (those with stable overnight oximetry capped) and speaking valve in most patients with OSA.

There are many opportunities to improve care for patients with OSA (whether known or unrecognized). Diagnosing and treating patients with OSA would likely prevent many cases of respiratory failure, and likely shorten the duration of mechanical ventilation.

Severe OSA increases the risk of stroke, heart attack, and mortality; treatment with CPAP effectively reduces that risk. ${ }^{4}$ There is convincing evidence that the majority of stroke patients have OSA, ${ }^{5}$ and that early treatment with CPAP improves recovery from stroke ${ }^{6}$ and reduces length of hospitalization. ${ }^{7}$ Could the same be true of patients with prolonged mechanical ventilation? Further studies are needed to assess the incidence of OSA among ventilator dependent patients, and to help determine whether early and optimal management for OSA improves outcome.

Douglas C Johnson MD Spaulding Rehabilitation Hospital Boston, Massachusetts

Karin Gardner Johnson MD Neurology Department Baystate Medical Center Springfield, Massachusetts

\section{REFERENCES}

1. MacIntyre NR, Epstein SK, Carson S, et al. Management of patients requiring prolonged mechanical ventilation: report of a NAMDRC consensus conference. Chest 2005;128(6):3937-3954.

2. Diaz-Abad M, Verceles AC, Brown JE, Scharf SM. Sleep-disordered breathing may be under-recognized in patients who wean from prolonged mechanical ventilation. Respir Care 2012;57(2):229-237.

3. Johnson DC, Campbell SL, Rabkin JD. Tracheostomy tube manometry: evaluation of speaking valves, capping and need for downsizing. Clin Respir J 2009;3(1):8-14. 


\section{Obstructive Sleep Apnea and Prolonged Mechanical Ventilation}

4. Bradley TD, Floras JS. Obstructive sleep apnea and its cardiovascular consequences. Lancet 2009;373(9657):82-93.

The authors have disclosed no conflicts of interest.

Correspondence: Douglas C Johnson MD, Spaulding Rehabilitation Hospital, 125 Nashua Street, Boston MA 02114. E-mail: djohnson5@partners.org.

DOI: $10.4187 /$ respcare. 01723
5. Johnson KG, Johnson DC. Frequency of sleep apnea in stroke and TIA patients: a meta-analysis. J Clin Sleep Med 2010;6(2): 131-137.

6. Bravata DM, Concato J, Fried T, Ranjbar N, Sandarangani T, McClain V, et al. Continuous positive airway pressure: evaluation of a novel therapy for patients with acute ischemic stroke. Sleep 2011; 34(9):1271-1277.

7. Kaneko Y, Hajek VE, Zivanovic V, Raboud J, Bradley TD et al. Relationship of sleep apnea to functional capacity and length of hospitalization following stroke. Sleep 2003;26(3):293-297. 